SUBJECT AREAS:

TARGETED THERAPIES

NANOPARTICLES

Received

26 August 2014

Accepted

28 November 2014

Published

15 December 2014

Correspondence and requests for materials should be addressed to L.X. (xulin83cn@gmail. com) or R.Y. (yinhero001@126. com)

* These authors contributed equally to this work.

\section{Enhanced cytotoxic activity of cetuximab in EGFR-positive lung cancer by conjugating with gold nanoparticles}

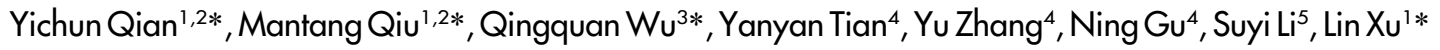
\& Rong Yin ${ }^{1 *}$

'Department of Thoracic Surgery, Jiangsu Key Laboratory of Molecular and Translational Cancer Research, Nanjing Medical University Affiliated Cancer Hospital, Cancer Institute of Jiangsu Province, Nanjing, P.R. China 210009 , ${ }^{2}$ The First Clinical College, Nanjing Medical University, Nanjing, P.R. China 210029, ${ }^{3}$ Department of Thoracic Surgery, Huai'an First People's Hospital, Nanjing Medical University, Huai'an, P. R. China 223300, ${ }^{4}$ State Key Laboratory of Bioelectronics, Jiangsu Key Laboratory for Biomaterials and Devices, School of Biological Science and Medical Engineering, Southeast University, Nanjing, P.R. China 210096, ${ }^{5}$ Department of Oncology, Southeast University Affiliated Zhongda Hospital, Nanjing, P.R. China 210009.

Cetuximab (C225) is a unique agent, targeting epidermal growth factor receptor (EGFR)-positive cancer. However, the therapeutic effect of C225 in EGFR high-expressing non-small cell lung cancer (NSCLC) remains poor. Here, we report that conjugation of C225 with gold nanoparticles (AuNPs) enhances the cytotoxicity of C225 in NSCLC both in vitro and in vivo. The NSCLC cell lines A549 (EGFR ${ }^{\text {high }}$ ) and H1299 $\left(\mathrm{EGFR}^{\mathrm{low}}\right)$ were employed to investigate different responses to C225, IgG-AuNPs and C225-AuNPs. The antitumor properties of C225-AuNPs were explored in vivo by establishing a tumor xenograft model in nude mice. Overall, the therapeutic effect of C225-AuNPs was more pronounced in EGFR high A549 cells compared with EGFR ${ }^{\text {low }} \mathrm{H} 1299$ cells. The cytotoxic effect of C225-AuNPs in A549 cells increased in a dose-dependent manner. C225-AuNPs significantly suppressed A549 cell proliferation and migration capacity and accelerated apoptosis compared with C225, and this effect was probably due to enhanced EGFR endocytosis and the subsequent suppression of downstream signaling pathway. Finally in the tumor xenograft of nude mice, treatment with C225-AuNPs also led to a significant reduction in tumor weight and volume with low toxicity. Our findings suggest that C225-AuNPs conjugate has promising potential for targeted therapy of EGFR positive NSCLC patients.

$\mathrm{N}$ anotechnology is widely used in interdisciplinary spanning the fields of chemistry, biology and medicine, with recent advances in the research here of oncotherapy ${ }^{1}$. Among all nanomaterials, gold nanoparticle (AuNP) is an attractive candidate for targeted delivery of various cancer therapeutic agents ${ }^{1,2}$. AuNPs are an ideal drug-delivery scaffold because of their distinctive features, including relatively high biocompatibility and facile conjugation to biomolecules and the unique optical properties conferred by their localized surface plasmon (LSP) resonance, which increases the ability to bind amine and thiol groups, allowing surface modification. Given these advantages, AuNPs have been successfully used to deliver a variety of anticancer therapeutics, in addition to their own theranostic applications ${ }^{3-5}$.

In recent years, the focus of oncology has moved towards targeted therapy $y^{6-7}$. Several molecular alterations have been viewed as potential therapeutic targets. Among these, the epidermal growth factor receptor (EGFR) is a particular hot topic in cancer treatment, and may be uniquely targeted using the monoclonal antibody, cetuximab (C225). C225 inhibits signal transduction by binding to the external domain of EGFR, thereby blocking ligand binding ${ }^{8-10}$. Besides colorectal cancer and head and neck cancer, NSCLC is the third major cancer type for which cetuximab has been evaluated. Because EGFR mutations in NSCLC are associated with chemosensitivity to gefitinib but not to cetuximab, it is speculated that cetuximab is effective against NSCLC, irrespective of EGFR mutation status ${ }^{6,8-9}$. Cetuximab has been used for the treatment of EGFR-expressing NSCLC in phase II and III trials, predominantly in combination with chemotherapy or radiotherapy. However, the overall response rate to cetuximab monotherapy is disappointingly low ${ }^{10-12}$.

AuNP-based therapy has been developed as a novel potential strategy in diagnosis and therapy, as drug delivery vehicles, contrast agents and radiation enhancers ${ }^{13-16}$. Previous studies have shown that AuNP-conjugated drugs may significantly increase chemosensitivity and delivery efficacy in several cancers, including pancreatic cancer 
and prostate cancer ${ }^{2,5}$. Based on this, it is highly possible that AuNPs may increase the sensitivity of NSCLC to C225. Thus, we synthesized C225-tethered AuNPs and investigated whether this compound could increase chemosensitivity to NSCLC both in cell lines and nude mice.

\section{Results}

Characterization of C225-AuNPs. The physical characteristics of C225-AuNPs and IgG-AuNPs were summarized in Table 1. The properties of C225-conjugated AuNPs were determined by TEM, zeta potential measurement, and Dynamic light scattering. TEM images reveal that unmodified AuNPs are monodispersed with average size of $14 \mathrm{~nm}$ (Fig. 1A \& 1B). From the dynamic light scattering (DLS) measurement, the hydrodynamic size of C225AuNPs is estimated to be $25 \mathrm{~nm}$ after BSA blocking, which is larger than the unmodified AuNPs (about $14 \mathrm{~nm}$ ) due to the contribution of protein adsorption layer (Fig. 1C). Zeta potential measurements show that surface potential of the unconjugated AuNPs is negatively charged with approximately $-42.7 \mathrm{mV}$. When coated with $\mathrm{C} 225$, the zeta potential increase to $-20.4 \mathrm{mV}$, demonstrating successful conjugation. With respect to the coupling ratio between AuNPs and C225, $13.97 \mu \mathrm{g} / \mathrm{ml} \mathrm{C225}$ were conjugated to $1 \mathrm{ml}$ AuNPs $(14 \mathrm{~nm}, 47.8 \mu \mathrm{g} / \mathrm{ml})$ detecting by BCA protein detection kits, so we can further calculate the number of coupling ratio through the calculation of the following methods. First, we calculated the number of AuNPs: $\mathrm{N}_{\text {AuNPs }}=47.8 \mu \mathrm{g} /(\mathrm{V} \times \rho)=$ $1.72 \times 10^{15}\left(\mathrm{~d}_{\text {AuNPs }}=14 \mathrm{~nm}, \mathrm{~V}_{\text {AuNPs }}=\pi \mathrm{d}^{3} / 6=1.436 \times 10\right.$ $\left.24 \mathrm{~m}^{3}, \rho_{\text {AuNPs }}=1.932 \times 10^{4} \mathrm{~kg} / \mathrm{m}^{3}\right)$, the molecular weight of $\mathrm{C} 225$ is $145.5 \mathrm{~kg} / \mathrm{mol}$, so we can get the number of C225: $\mathrm{N}_{\mathrm{C} 225}=$ $13.97 \mu \mathrm{g} / \mathrm{M} * \mathrm{NA}=5.78 \times 10^{16}$, The conjugation number of $\mathrm{C} 225$ molecules per gold nanoparticle was determined to be 34 $\left(\mathrm{N}_{\mathrm{C} 225}: \mathrm{N}_{\mathrm{AuNPs}} \approx 34\right)$.

Effect of C225-AuNPs on cell cytotoxicity. To test the effect of C225-AuNPs on the growth of NSCLC cell lines, we employed the CCK-8 assay and clonogenic assay (Fig. 2, 3).Previous studies have demonstrated that AuNPs are nontoxic ${ }^{1,13}$. In accordance, we also observed that cell proliferation was not apparently affected both in A549 and H1299 cell lines following treatment with IgG-AuNPs. On the other hand, C225-AuNPs significantly inhibited cell proliferation in the EGFR high-expressing A549 cell line, in a time- and dosedependent manner, compared with $\mathrm{C} 225$ alone, with inhibition peaking at $500 \mu \mathrm{g} / \mathrm{mL}$ at $48 \mathrm{~h}$ (cell inhibition ratio: $24 \mathrm{~h} 20.1 \%$ vs $11.7 \%$; 48 h $29.1 \%$ vs $12.0 \%$ ). In contrast, C225-AuNPs modestly increased cytotoxicity in the EGFR low-expressing H1299 cell line, compared with C225 alone. Clonogenic assay showed similar conclusion. In A549 cell, the colonies in C225-AuNPs $(100 \mu \mathrm{g} / \mathrm{mL})$ group was fewer than $\mathrm{C} 225$ and IgG-C225 group. While, there is no significant difference in colony counts between three treated groups in H1299 cells. These results demonstrate that C225-AuNPs are capable of increasing chemosensitivity, particularly in EGFR high-expressing NSCLC cells.

Cell apoptosis and cell cycle analysis in C225-AuNPs treated cells. We next analyzed whether C225-AuNPs inhibited cell proliferation by inducing apoptosis. Treatment of A549 cells with C225-AuNPs $(100 \mu \mathrm{g} / \mathrm{mL})$ led to a significant increase in apoptosis compared with

Table 1 | Physical characteristics of C225-AuNPs and lgG-AuNPs

\begin{tabular}{lll} 
& C225-AuNPs & lgG-AuNPs \\
\hline size & $25 \mathrm{~nm}$ & $30 \mathrm{~nm}$ \\
charge & $-20.4 \mathrm{mV}$ & $-18.1 \mathrm{mV}$ \\
polydispersity index & 0.463 & 0.334 \\
coupling ratio & $34: 1$ & $39: 1$ \\
\hline
\end{tabular}
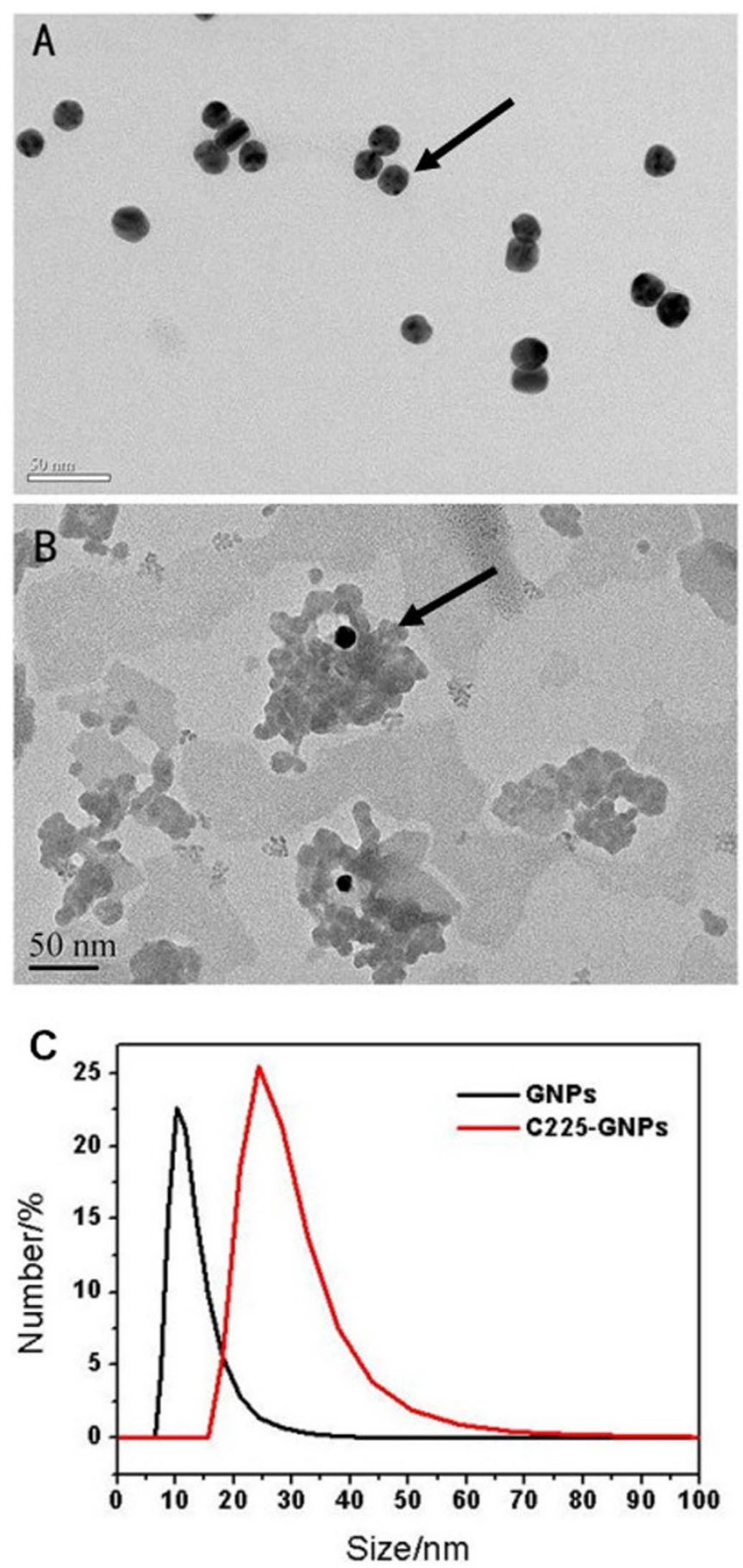

Figure $1 \mid$ Representative transmission electron microscope (TEM) images of (A) bare gold nanoparticles (AuNPs) with an average diameter of $14 \mathrm{~nm}$ and (B) Cetuximab-conjugated gold nanoparticles (C225-AuNPs). (C) The hydrodynamic size of AuNPs and C225-AuNPs measured by dynamic light scattering (DLS).

treatment of cells with $\mathrm{C} 225$ at the same concentration $(40.2 \pm 4.03 \%$ vs. $17.0 \pm 2.67 \%, p<0.01$, Fig. $4 \mathrm{~A}$ ). In contrast, we observed no significant difference in the rate of apoptosis in H1299 cells following treatment with C225, IgG-AuNPs or C225-AuNPs at doses ranging between 1-100 mg/L (Fig. 4B). We also assessed the effect of these compounds on cell cycle. As shown in Fig. 5, treatment of A549 or H1299 cells with C225-AuNPs did not lead to a G1/G0 phase delay in either cell line compared with treatment with C225 and IgG-AuNPs. Taken together, these results suggest that C225-AuNPs induced cell apoptosis without altering cell cycle distribution. 
A549(24h)

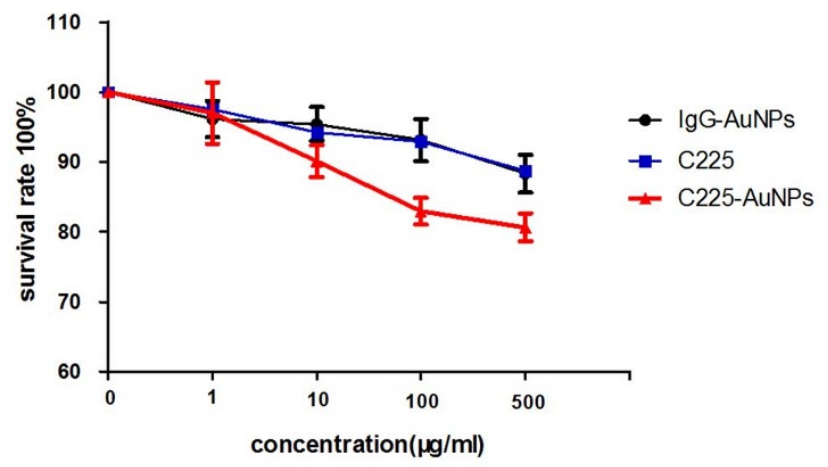

H1299(24h)

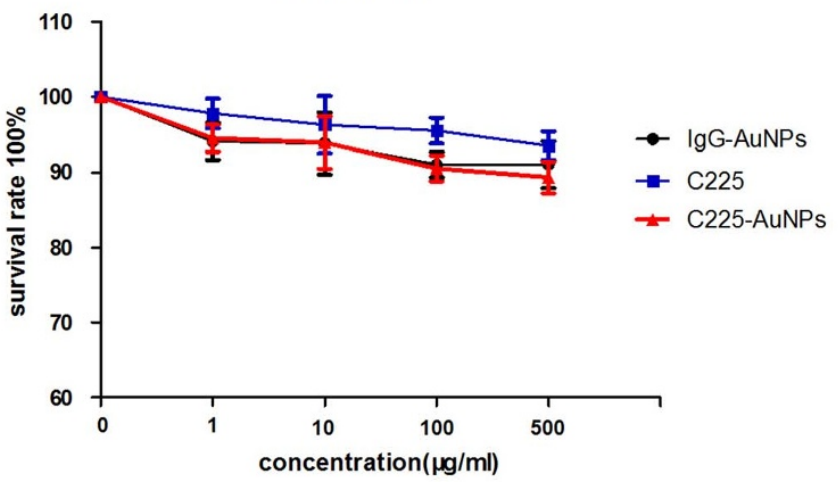

A549(48h)

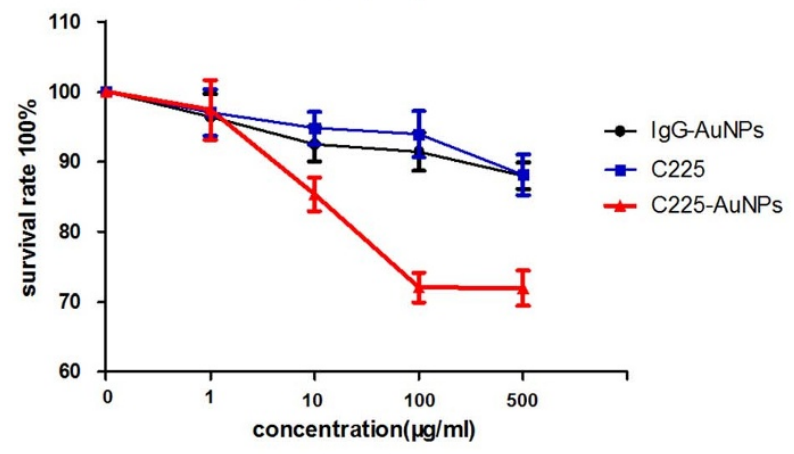

H1299(48h)

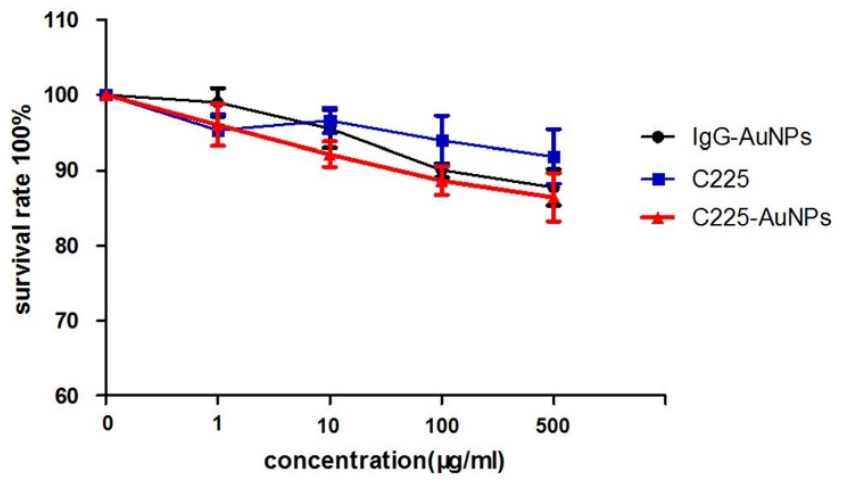

Figure 2 Cytotoxicity of A549 and H1299 cells incubated with C225, IgG-AuNPs or C225-AuNPs for 24 h and 48 h at various concentrations (1, 10, $100,500 \mu \mathrm{g} / \mathrm{ml})$. Data represents mean $\pm \mathrm{SD}, \mathrm{n}=6$.

C225-AuNPs inhibit the migration of A549 cells. We next performed transwell migration assays to evaluate the effects of C225-AuNPs on migration. As shown in Fig. 6, Both in A549 and H1299 cells, the three treatments significantly impaired migration at $100 \mu \mathrm{g} / \mathrm{ml}$, however treatment of A549 cells with C225-AuNPs $(100 \mu \mathrm{g} / \mathrm{mL})$ apparently inhibited cell migration compared with C225 and IgG-AuNPs treatment groups (mean cell number, $94 \pm 9.1$ vs. $155 \pm 2.2$ and $136 \pm 15.7$, respectively, Fig. 6A).Conversely, no significant differences were observed following treatment of H1299 cells with C225-AuNPs compared with C225 and IgG-AuNPs (mean cell number $152 \pm 3.0$ vs. $160 \pm 8.1$ and $166 \pm 7.5$, respectively, Fig. $6 \mathrm{~B}$ ).

Influence of C225-AuNPs on the EGFR signaling pathway in A549 cells. To explore whether C225-AuNPs exert anti-cancer effect by affecting EGFR signaling, EGFR protein itself, as well as the downstream protein levels were examined by western blot in A549 cells. Because C225 is an EGFR-targeting monoclonal antibody, we reasoned that EGFR levels may be suppressed in the C225 alone treatment group. However, we surprisingly found total EGFR level in C225-AuNPs group were not significantly inhibited, but even higher than the C225 and IgG-AuNPs groups (Fig. 7A \& B). Since only the extracellular domain of membranous EGFR could be activated by its ligand, we therefore further detected the expression of membranous and cytoplasmic EGFR respectively. As shown in Fig. 7C \& D, the membranous EGFR level was significantly downregulated, whereas the cytoplasmic EGFR level was dramatically higher than the other two treatment groups. These data suggest that C225-AuNPs might block the EGFR signaling pathway via enhancing the EGFR endocytosis and inducing the accumulation of cytoplasmic inactivated EGFR. Finally, the downstream key protein of EGFR pathway, including Akt, Erk, and Bcl-2 were detected by WB and displayed in Fig. 7A \& B. Reasonably, proliferation related p-Akt and p-Erk levels were both significantly down-regulated, and apoptosis related Bcl-2 level was relatively up-regulated in C225-AuNPs group as compared with C225 and IgG-AuNPs groups. Taken together, our results demonstrate that C225-AuNPs could suppress the EGFR signaling pathway probably via inducing membranous EGFR endocytosis and cytoplasmic EGFR accumulation.

C225-AuNPs inhibit tumor growth in a NSCLC xenograft nude mouse model. To investigate whether C225-AuNPs could inhibit NSCLC tumor growth in vivo, nude mice were subcutaneously inoculated with A549 and H1299 cells. There was no significant weight lost observed in both C225-AuNPs and IgG-AuNPs group (Fig. 8A \& B), which means the nano-conjugates were low toxic. Tumor growth curves of A549 and H1299 tumor-bearing mice with different treatment were shown in Fig. 8C \& D. We observed a significant decrease in A549 tumor volume in 21-day C225AuNPs-treated mice compared with IgG-AuNPs and C225 treatment groups $\left(309.24 \pm 55.98 \mathrm{~mm}^{3}\right.$ vs. $436.55 \pm 36.65 \mathrm{~mm}^{3}$ and $473.65 \pm 60.43 \mathrm{~mm}^{3}$, respectively, $p<0.05$ ). In contrast, this effect was not as pronounced in H1299 tumor bearing mice following treatment with C225-AuNPs for 21 days $\left(408.88 \pm 39.91 \mathrm{~mm}^{3}\right.$ vs. $448.44 \pm 79.00 \mathrm{~mm}^{3}$ and $374.37 \pm 67.06 \mathrm{~mm}^{3}$, respectively). Tumor weight was further confirmed at the end of the experiment after sacrificing mice. In A549 tumors, treatment with C225-AuNPs showed the largest inhibitory effect among all groups, with an average tumor mass of $0.28 \pm 0.09 \mathrm{~g}$, compared with $0.51 \pm$ $0.23 \mathrm{~g}$ for PS-treated mice and $0.40 \pm 0.11 \mathrm{~g}$ for C225-treated mice (Fig. 8E). By comparison, no statistical differences in tumor mass were observed between various treatment groups in H1299 tumors. These data indicate that C225-AuNPs may inhibit lung tumor growth in vivo, specifically in EGFR high-expressing NSCLC. 
A549
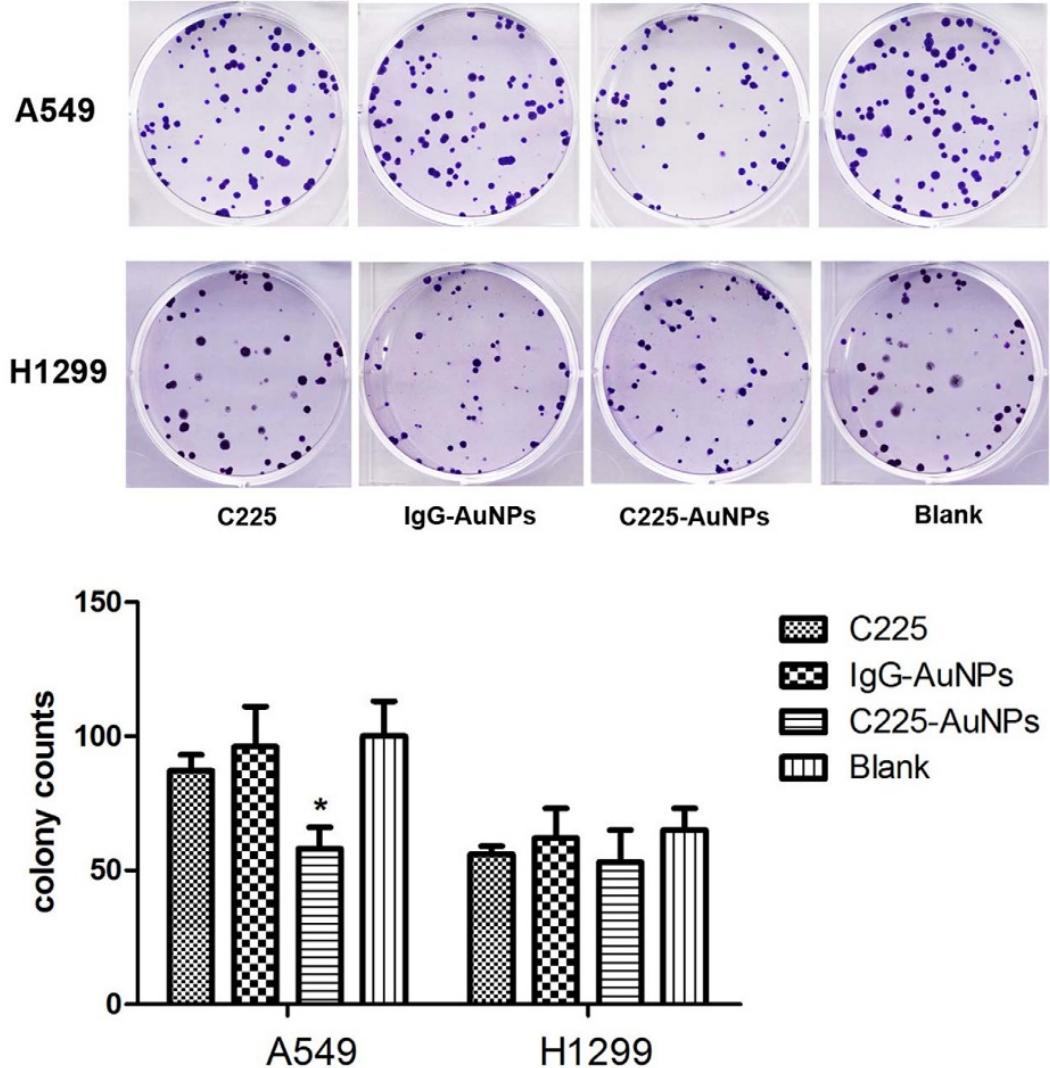

$\mathrm{C} 225$

$\infty$ IgG-AuNPs

○ C225-AuNPs

孟 Blank

A549

$\mathrm{H} 1299$

Figure $3 \mid$ The colony formation of A549 and H1299 cells treated with C225, IgG-AuNPs or C225-AuNPs at $100 \mu \mathrm{g} / \mathrm{ml}$. Values represent the means \pm SD. $(* * p<0.01 ; * p<0.05)$.

\section{Discussion}

The overexpression of EGFR in NSCLC is associated with tumor development, poor prognosis and drug resistance ${ }^{17,18}$. Cetuximab is the first monoclonal antibody authorized by the FDA for EGFRtargeted therapy, however, cetuximab has limited effects in EGFRpositive NSCLC patients. Indeed, several phase III clinical trials revealed that cetuximab did not significantly improve clinical outcome in NSCLC patients ${ }^{6,8,19-20}$. Brown et al. ${ }^{21}$ developed a drugdelivery strategy for platinum-based anticancer drugs based on AuNPs, which offer a new approach to cancer therapy delivery. Here, we establish a novel compound (C225-AuNPs) by conjugating gold nanoparticles to C225. This compound displays a stronger
A
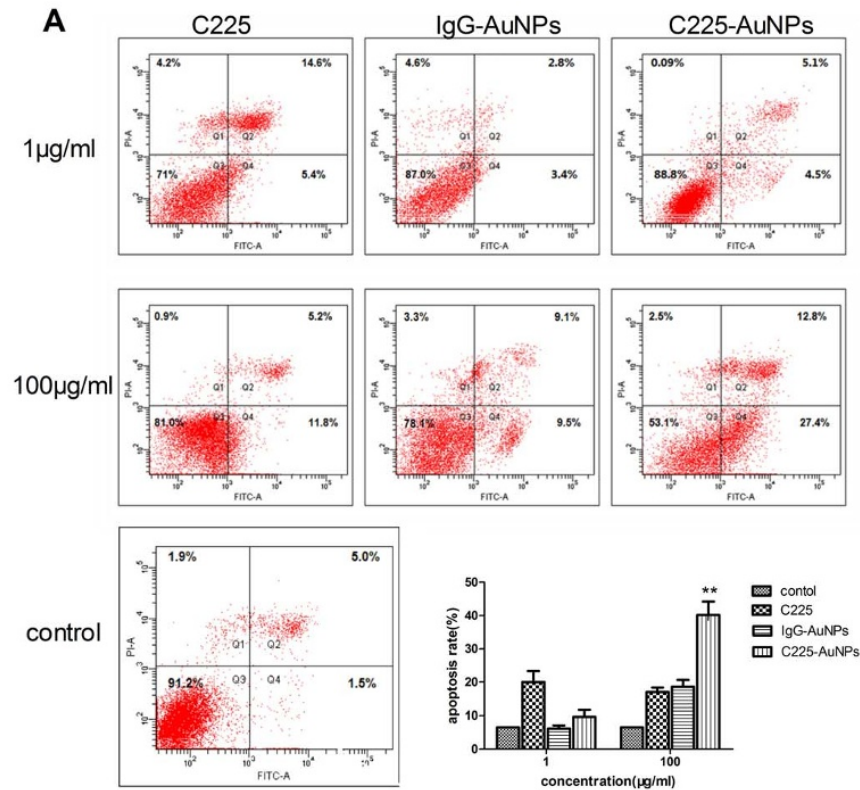

B
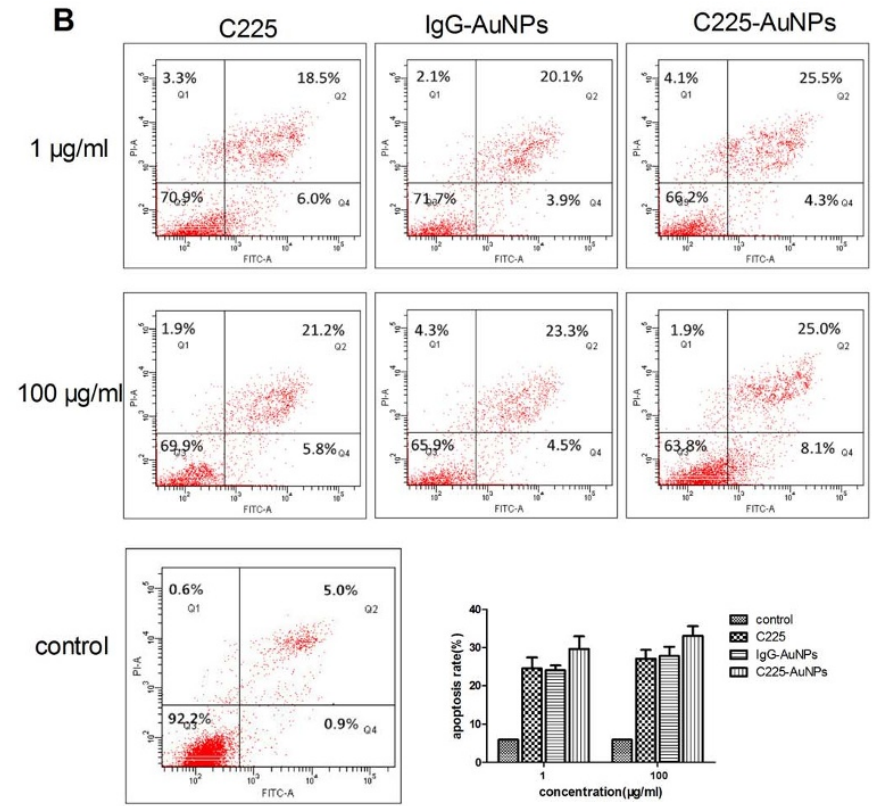

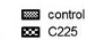
吕 $\lg$ G-AuNPs
C225-AuNPs

Figure $4 \mid$ Apoptotic evaluation of cell lines treated with indicated concentrations of C225, IgG-AuNPs or C225-AuNPs for 48 h using the FITCAnnexin V apoptosis Detection Kit I assay. A) A549 cells; B) H1299 cells. Values represent the means \pm SD. $\left(* * p<0.01 ;{ }^{*} p<0.05\right)$. 

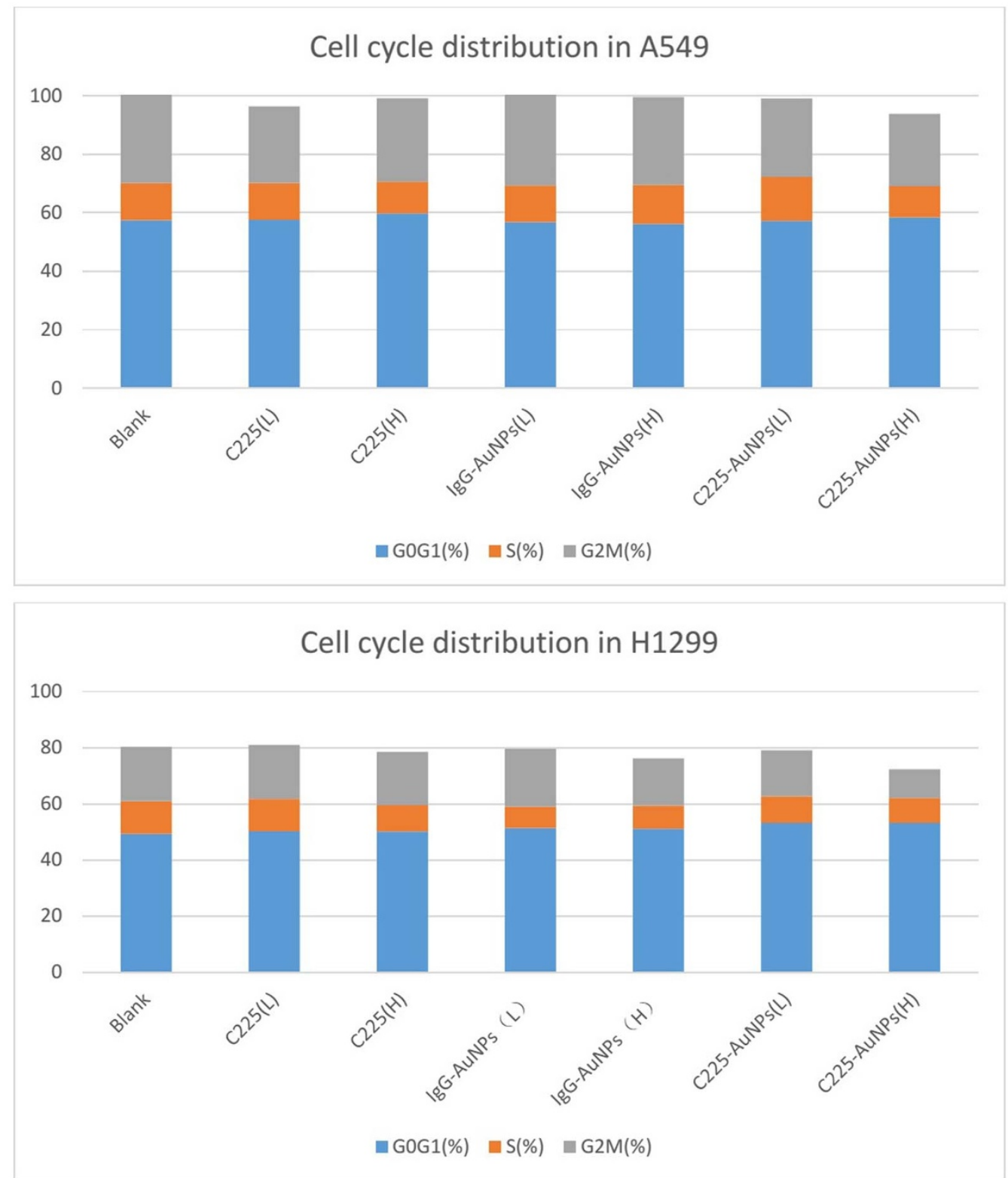

Figure 5 | Cell cycle distribution of cell lines treated with indicated concentrations of C225, IgG-AuNPs or C225-AuNPs.
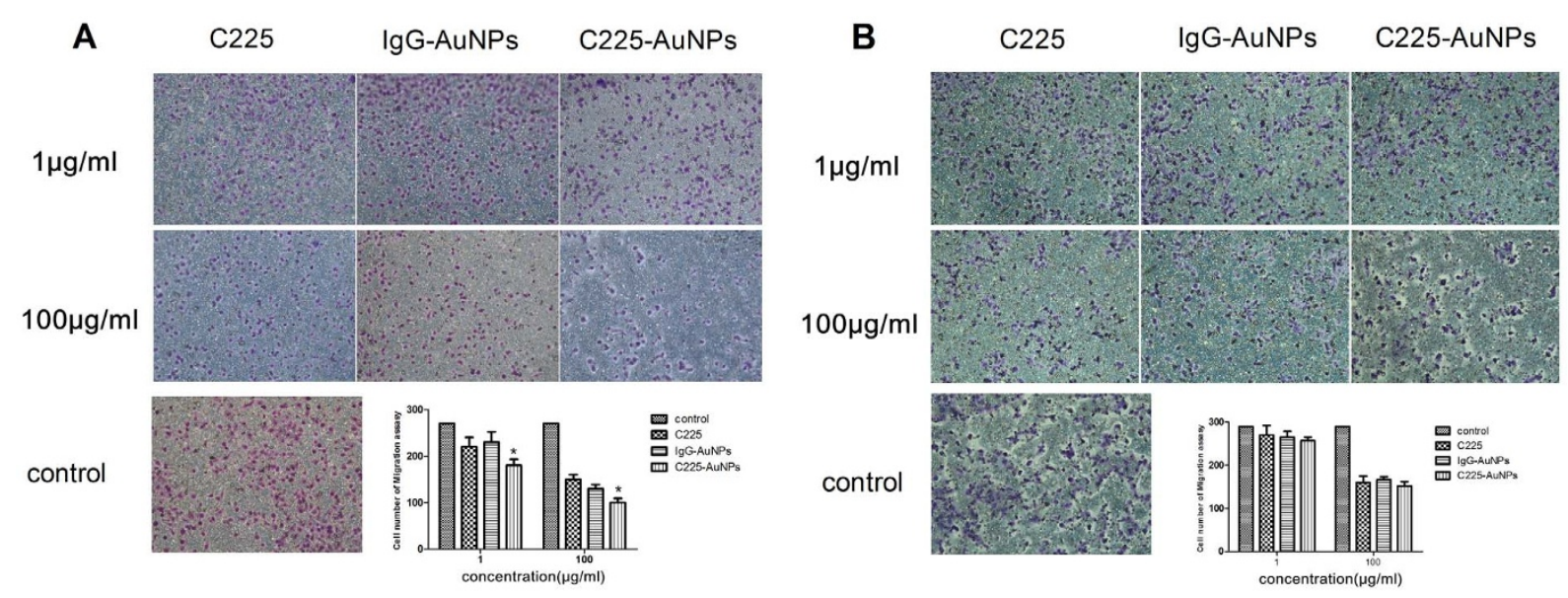

Figure 6 Inhibitory effect of C225, IgG-AuNPs or C225-AuNPs on cell migration in (A): A549 cells; (B): H1299 cells. Values represent the mean \pm SD. $\left({ }^{* *} \mathrm{p}<0.01 ; * \mathrm{p}<0.05\right)$. 
A

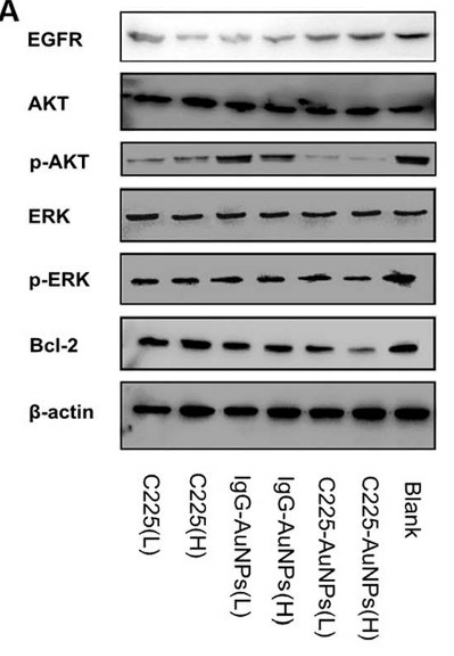

B
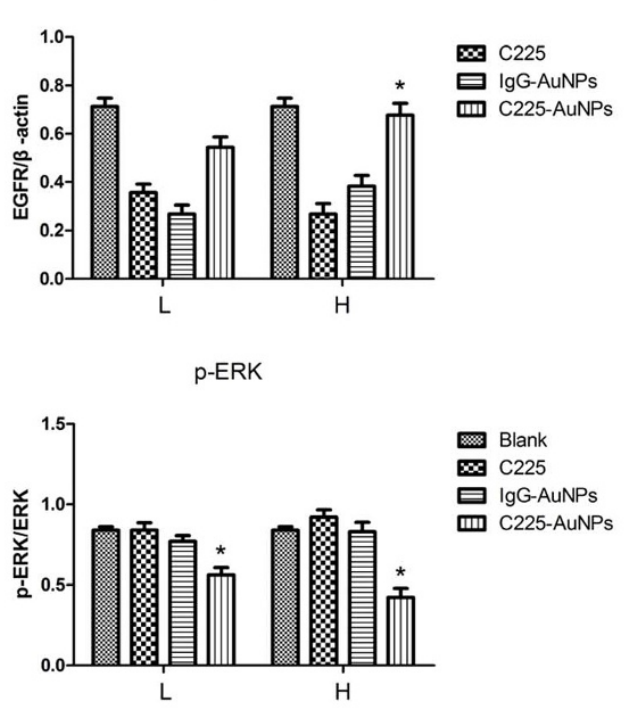

D

C
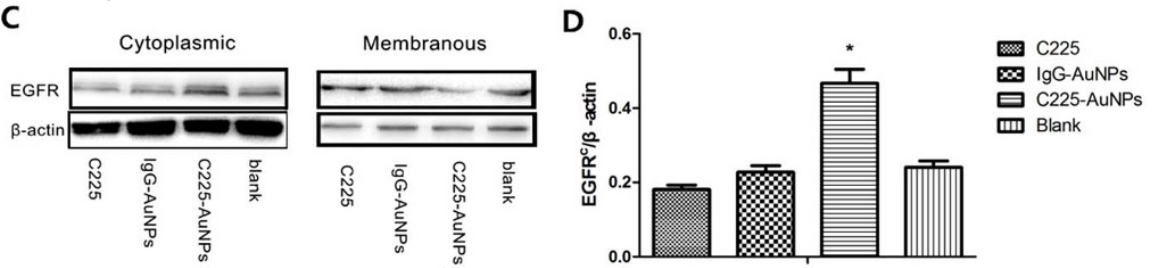

p-AKT

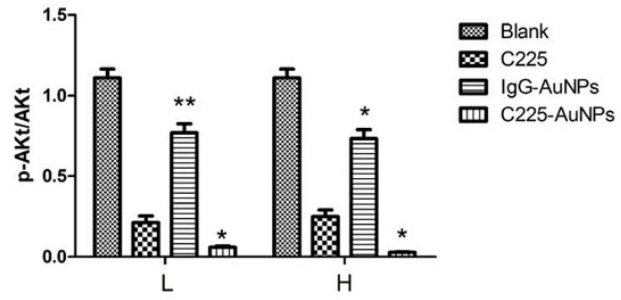

$\mathrm{Bcl}-2$

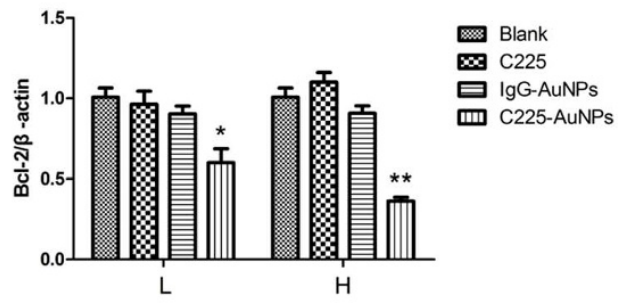

Figure $7 \mid$ (A), (B): Western blot analysis of C225, IgG-AuNPs or C225-AuNPs on levels of EGFR, Akt, phospho-Akt, ERK, phospho-ERK and Bcl-2 in EGFR-high expressing A549 cells. (C), (D): Western blot analysis of C225, IgG-AuNPs or C225-AuNPs on levels of membranous and cytoplasmic EGFR in A549 cells. $\beta$-actin serves as a loading control. The results shown are representative of three independent experiments. Values represent the mean \pm SD. $\left({ }^{* *} \mathrm{p}<0.01 ;{ }^{*} \mathrm{p}<0.05\right)$.

toxicity profile compared with free, unconjugated C225 in vitro and in vivo, indicating that C225-AuNPs have sufficient antitumor activity to inhibit tumor growth.

The biological characteristics of AuNPs, such as cytotoxicity and cellular uptake, are depended on surface size to a large extent. Pan et $a^{22}$ demonstrated that gold nanoparticles with a diameter of $1.4 \mathrm{~nm}$ can induce cell death by oxidative stress, whereas $15 \mathrm{~nm}$ gold nano- particles exhibit minimal cytotoxicity. Secondly, the AuNPs with $50 \mathrm{~nm}$ diameter present unique advantages over other nanoparticles in terms of cellular uptake, and 15 to $20 \mathrm{~nm}$ AuNPs also have relatively stronger endocytosis capacity ${ }^{4}$. Finally, the binding efficiency with C225 is also size-dependent, and the AuNPs with a relatively larger size, like 15-30 nm, usually have better binding affinity with C225 (our preliminary data not shown). Taken together, in order to
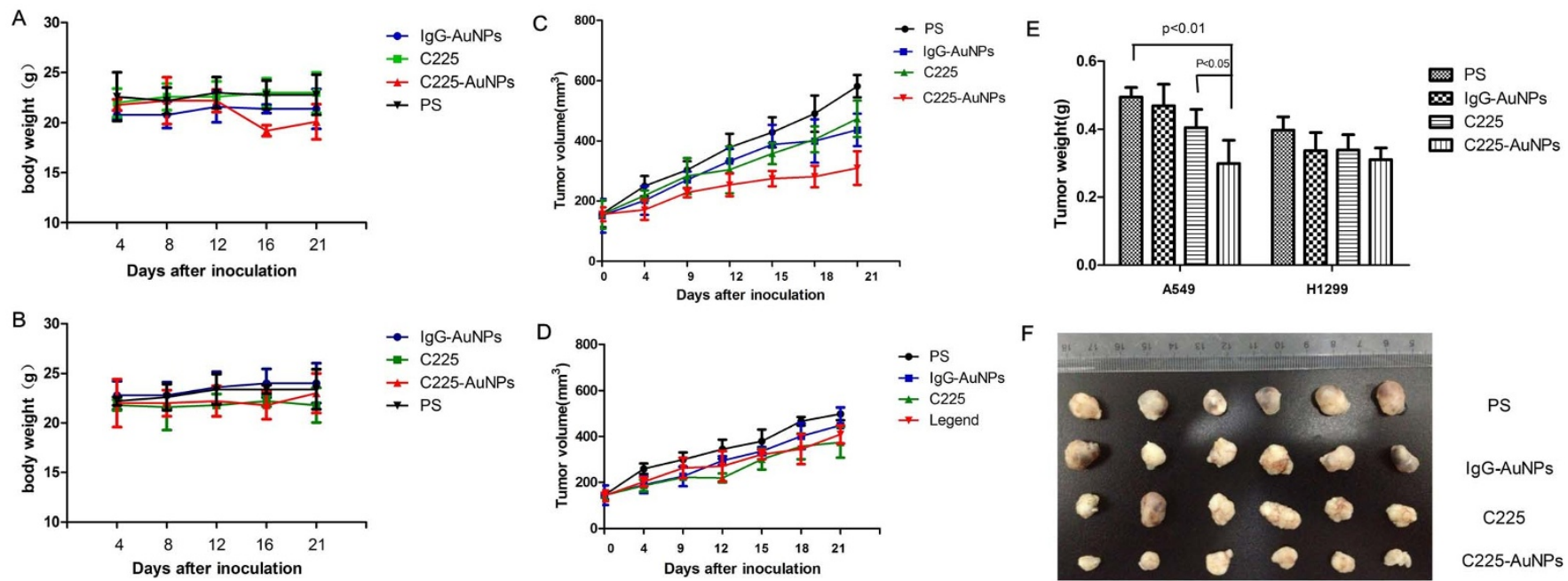

PS

IgG-AuNPs

$\mathrm{C} 225$

C225-AuNPs

Figure 8 Antitumor effects of IgG-AuNPs, C225 and C225-AuNPs in a mouse lung cancer model. (A): Body weight of A549 tumor-bearing mice treated with physiologic saline (PS), C225, IgG-AuNPs and C225-AuNPs. (B): Body weight of H1299 tumor-bearing mice (C): Tumor growth curves of A549 tumor-bearing mice. (D): Tumor growth curves of H1299 tumor-bearing mice; (E): Individual tumor weights from four groups of mice; (F): Images of tumors from four groups after sacrificing mice at day 21 . Data are expressed as the mean $\pm \operatorname{SD}(\mathrm{n}=6) .\left({ }^{* *} \mathrm{p}<0.01 ;{ }^{*} \mathrm{p}<0.05\right)$. 
avoid interrupting the anti-cancer effects of C225 by AuNPs alone, and to gain better endocytosis and binding efficiency with C225, the AuNPs with a diameter of about $14 \mathrm{~nm}$ were therefore chosen in our study. As we expected, C225 modified AuNPs displayed a significantly elevated gathering especially in EGFR ${ }^{\text {high }}$ A549 cells. Besides the specificity on targeting EGFR of C225 itself, we speculated that the endocytosis capacity achieved by AuNPs can increase the intracellular concentrations of $\mathrm{C} 225$ in cancer cells, compared with the use of free drugs ${ }^{23}$.

Then we focus on the anti-tumor property of C225-AuNPs in vitro and in vivo. C225-AuNPs significantly inhibit cell proliferation, weaken the migration, simultaneous accelerate C225-induced apoptosis without affecting cell cycle arrest. In mice models, our results also suggest that C225-AuNPs can conspicuously inhibit tumor growth, the main mechanism is probably associated with the larger intracellular amount of C225, which is benefit by the internalized AuNPs. It was also proven that AuNPs can bind to VEGF and then inhibit cell angiogenic activity, which is a key procedure involved in tumor formation ${ }^{24,25}$.

The results of our study indicate that C225-AuNPs are more effective in A549 cells, which express high levels of EGFR. The ERK/ MAPK and PI3K/Akt signaling pathways are two major signaling pathways that lie downstream of EGFR. These pathways are distinctly associated with cell proliferation, apoptosis and differentiation processes in $\mathrm{NSCLC}^{11,24}$. Alteration of AKT and ERK following treatment of A549 cells with C225 was insignificant, even at a high concentrations. In contrast, treatment of A549 cells with C225-AuNPs led to significant inhibition of both AKT and ERK phosphorylation. Furthermore, down-regulation of Bcl-2 was observed in C225-AuNPs treated cells, suggesting that C225AuNPs are intimately involved in the process of cell apoptosis. However, EGFR expression seemed to be "rescued" by C225 conjugated nanoparticles when compared with C225 alone and IgGAuNPs, In order to discover the underlying mechanism, we further detected the expression of membranous and cytoplasmic EGFR respectively. We found C225-AuNPs significantly decreased the membranous EGFR level and dramatically increased the cytoplasmic inactivated EGFR level comparing with C225 alone (Fig. 7). Recently, it has been reported that C225-conjugated AuNPs could enhance EGFR endocytosis and alert its intracellular trafficking in pancreatic cancer cells ${ }^{26}$. Therefore, based on our data, we have reason to believe that C225-conjugates could suppress the EGFR signaling pathway probably via inducing membranous EGFR endocytosis and cytoplasmic EGFR accumulation.

In summary, we describe a safe and efficient drug delivery device for target therapy of lung cancer based on $14 \mathrm{~nm}$ gold nanoparticles. More importantly, our present data demonstrates that conjugation of C225 to $14 \mathrm{~nm}$ gold nanoparticles efficiently promotes cytotoxicity and antitumor capability in EGFR positive NSCLC cells than C225 alone in vitro and in vivo, which undoubtedly provides a potential strategy for clinical NSCLC targeted therapy.

\section{Methods}

Synthesis and characterization of C225-AuNPs. First, Gold nanoparticles (AuNPs) were synthesized from tetrachlorauric acid $\left(\mathrm{HAuCl}_{4}\right)$ by wet chemical methods using trisodium citrate as a reducing agent. Briefly, water solutions of $\mathrm{HAuCl}_{4}$ and trisodium citrate were mixed under heavy boiling, resulting in gold particles with a net negative charge from the adsorption of citrate ions, which stabilized the particles. Varying the citrate to $\mathrm{HAuCl}_{4}$ concentration ratio produced particles with an average size of about $14 \mathrm{~nm}$. The $\mathrm{pH}$ of the AuNP solution was increased to approximately 8.5 by potassium carbonate, which is the isoelectric point of $\mathrm{C} 225$. A total of $100 \mu \mathrm{g}$ of C225 was added slowly to $2 \mathrm{~mL} \mathrm{pH}$-adjusted AuNP solution and was placed on a continuous mixer and incubated at ambient temperature for $1 \mathrm{~h}$. After that, blocking treatment was performed by using BSA (bovine serum albumin) at a final concentration of $1 \%(\mathrm{w} / \mathrm{w})$. Then the solution was centrifuged at 10,000 RPM for $40 \mathrm{~min}$ to remove unbound antibodies. AuNPs and C225-AuNPs were subsequently observed by transmission electron microscope (TEM). The amount of conjugated C225 antibody was determined by the bicinchoninic acid (BCA) method. Dynamic light scattering(DLS) and zeta potential experiments were conducted in a Zetasizer instrument (Nano ZS, Malvern Instruments Ltd). The particle size and zeta potential were measured simultaneously three times and in triplicate.

Human lung cancer cell lines. The A549 human lung adenocarcinoma cell line, which expresses high levels of wild type EGFR, and the low-EGFR expressing H1299 cell line were utilized for all experiments. A549 and H1299 cell lines were purchased from the Institute of Biochemistry and Cell Biology, Chinese Academy of Science. Cells were maintained in RPMI-1640 culture media (Gibco, NY, USA) supplemented with $10 \%$ fetal bovine serum (FBS, Gibco) at $37^{\circ} \mathrm{C}$ in a $5 \% \mathrm{CO}_{2}$ incubator.

Cytotoxicity assays. A549 and H1299 cells were seeded in 96-well plates $\left(6 \times 10^{3}\right.$ cells/well) in RPMI-1640 medium and incubated with C225, IgG-AuNPs or C225AuNPs at various concentrations $(1,10,100,500 \mu \mathrm{g} / \mathrm{mL})$ for $24 \mathrm{~h}$ or $48 \mathrm{~h}$ at $37^{\circ} \mathrm{C}$ in a $5 \% \mathrm{CO}_{2}$ incubator. Relative cell growth was determined using the Cell Counting Kit- 8 (CCK-8, Dojindo) in accordance with the manufacturer's instructions. The cell inhibition rate was calculated using the formula: cell inhibition rate $=\left(1-\mathrm{A}_{\text {Test }} /\right.$ $\left.\mathrm{A}_{\text {Control }}\right) / 100 \%$, where $\mathrm{A}_{\text {Test }}$ is the absorbance of the experimental wells and $\mathrm{A}_{\text {Control }}$ is the absorbance of the control well. All experiments were performed in biological triplicate.

Clonogenic assay. A549 and H1299 cells were trypsinized and plated in 6-well plates. Then the cells were treated with C225, IgG-AuNPs and C225-AuNPs $(100 \mu \mathrm{g} / \mathrm{mL})$, Plates were incubated to allow colony formation for 14 days. The colonies were fixed with methanol and stained with Giemsa. Finally, the plates were inspected by microscopy and the number of the colonies were counted. Each assay was made in triplicate and only colonies containing at least 50 cells were counted.

Flow cytometry. For analysis of apoptosis, A549 and H1299 cells were incubated with C225, IgG-AuNPs and C225-AuNPs $(1 \mu \mathrm{g} / \mathrm{mL}$ or $100 \mu \mathrm{g} / \mathrm{mL})$ for $48 \mathrm{~h}$. Cells were harvested from 6-well plates following trypsinization, washed gently with PBS $(\times 2)$ and collected by centrifugation. Cells were subsequently stained using the FITCAnnexin V Apoptosis Detection Kit I (BD Biosciences, NJ, USA) in accordance with the manufacturer's protocol. Cells were analyzed by flow cytometry (BD Biosciences). For cell cycle analysis, cells were treated with indicated concentrations of C225, IgGAuNPs and C225-AuNPs for $48 \mathrm{~h}$, harvested and resuspended in $70 \%$ ethanol at $4{ }^{\circ} \mathrm{C}$ for $2 \mathrm{~h}$. After washing with cold PBS $(\times 2)$, cells were treated with RNase $(0.25 \mathrm{mg}$ / $\mathrm{mL})$ at $37^{\circ} \mathrm{C}$ for $1 \mathrm{~h}$ and stained with propidium iodide $(50 \mu \mathrm{g} / \mathrm{mL})$ at $4^{\circ} \mathrm{C}$ for $30 \mathrm{~min}$. DNA content was analyzed by flow cytometry.

Migration assays. Cell migration assays were performed using a two-chambertranswell device with an $8 \mu \mathrm{m}$ - pore size membrane (Millipore, MA, USA). Cells were harvested as described above, and $5 \times 10^{4}$ cells were resuspended in $200 \mu \mathrm{l}$ RPMI1640 medium without FBS and seeded into the upper chamber of the device. RPMI1640 medium $(500 \mu \mathrm{l})$ containing $10 \%$ FBS was added to the lower chamber and the device was incubated at $37^{\circ} \mathrm{C}$ for $12 \mathrm{~h}$. Membranes were rinsed with PBS and stained with a $0.1 \%$ crystal violet staining solution for $30 \mathrm{~min}$. The inner side of the upper chamber was wiped with a cotton swab to remove the cells and after drying, four random fields were analyzed for each chamber. Assays were conducted in triplicate in three independent experiments.

Western blot. Total protein was extracted by lysing the cells in lysis buffer $(300 \mu \mathrm{l})$ containing protease inhibitors. The cytoplasmic and membranous proteins were isolated using a subcellular protein fractionation kit (Thermo Scientific, Rockford, IL) following the manufacturer's instructions. Lysate protein concentration was measured using a BCA protein assay kit. Protein extracts were subjected to sodium dodecyl sulfate-polyacrylamide gel electrophoresis and transferred to polyvinylidene difluoride membranes (Bio-Rad, CA, USA). Membranes were incubated with primary antibodies for EGFR, Akt, phosphoAkt, ERK, phospho-ERK, Bcl-2 or $\beta$-actin $(1: 1000$, Cell Signaling Technology, USA) overnight. After washing three times, the membranes were incubated with secondary antibody ( $1: 1000$, Abcam, USA) for $2 \mathrm{~h}$ at room temperature. Immunoreactive bands were visualized by chemiluminescence with Pierce ECL detection reagent (Millipore, MA, USA). Each experiment was performed at least three times, independently.

Xenograft model in nude mice. The study was approved by the Ethics Committee of Cancer Institute of Jiangsu Province. Care and treatment of experimental animals was performed in accordance with institutional guideline. All mice were housed and handled under specific, aseptic conditions and maintained on a 12-h light-dark cycle with appropriate temperature. Suspensions of A549 and $\mathrm{H} 1299$ cells $\left(5 \times 10^{6}\right)$ were injected subcutaneously into the backs of the mice. Tumor-bearing mice (tumor volume $\sim 70-100 \mathrm{~mm}^{3}$ ) were randomly separated into four groups ( $\mathrm{n}=12$ /group), including a physiologic saline (PS) control group and three treatment groups (C225, IgG-AuNPs and C225-AuNPs). C225, IgG-AuNPs and C225-AuNPs were injected by intravenous tail injection ( $4 \mathrm{mg} / \mathrm{kg}$, twice per week for three consecutive weeks). The control group was injected with PS alone. Mouse weight and tumor size was measured and tumor volumes were calculated as $0.5 \times$ length $\times(\text { width })^{2}$. Mice were sacrificed on day 21 and tumor weight was measured.

Statistical Analysis. All values are expressed as means \pm standard deviation. The statistical significance of differences was analyzed by Student's t-test and one-way 
analysis of variance, using the SPSS 20.0 statistical package. A $p$ value less than 0.05 was considered statistically significant.

1. Ahmad, M. Z. et al. Nanometric gold in cancer nanotechnology: current status and future prospect. J Pharm Pharmacol. 65, 634-651 (2013).

2. Joseph, M. M., Aravind, S. R., Varghese, S., Mini, S. \& Sreelekha, T. T. PST-Gold nanoparticle as an effective anticancer agent with immunomodulatory properties. Colloids Surf B Biointerfaces. 104, 32-39 (2013).

3. Kao, H. W. et al. Evaluation of EGFR-targeted radioimmuno-gold-nanoparticles as a theranostic agent in a tumor animal model. Bioorg Med Chem Lett. 23, 3180-3185 (2013).

4. Lan, M. Y. et al. Induction of apoptosis by high-dose gold nanoparticles in nasopharyngeal carcinoma cells. Auris Nasus Larynx. 40, 563-568 (2013).

5. Curley, S. A. et al. Noninvasive radiofrequency field-induced hyperthermic cytotoxicity in human cancer cells using cetuximab-targeted gold nanoparticles. J Exp Ther Oncol. 7, 313-326 (2008).

6. Pirker, R. EGFR-directed monoclonal antibodies in non-small cell lung cancer. Target Oncol. 8, 47-53 (2013).

7. Leung, L., Mok, T. S. \& Loong, H. Combining chemotherapy with epidermal growth factor receptor inhibition in advanced non-small cell lung cancer. Ther Adv Med Oncol. 4, 173-181 (2012).

8. Ryu, S. H. et al. Intracytoplasmic epidermal growth factor receptor shows poor response to the cetuximab antitumor effect in irradiated non-small cell lung cancer cell lines. Lung Cancer. 77, 482-487 (2012).

9. Khan, J. A. et al. Designing nanoconjugates to effectively target pancreatic cancer cells in vitro and in vivo. PLoS One. 6, e20347 (2011)

10. Khambata-Ford, S. et al. Analysis of potential predictive markers of cetuximab benefit in BMS099, a phase III study of cetuximab and first-line taxane/ carboplatin in advanced non-small-cell lung cancer. J Clin Oncol. 28, 918-927 (2010).

11. Krumbach, R. et al. Primary resistance to cetuximab in a panel of patient-derived tumour xenograft models: activation of MET as one mechanism for drug resistance. Eur J Cancer. 47, 1231-1243 (2011).

12. Hainfeld, J. F. et al. Micro-CT enables microlocalisation and quantification of Her2-targeted gold nanoparticles within tumour regions. Br J Radiol. 84, 526-533 (2011).

13. Webster, D. M., Sundaram, P. \& Byrne, M. E. Injectable nanomaterials for drug delivery: carriers, targeting moieties, and therapeutics. Eur J Pharm Biopharm. 84, $1-20$ (2013).

14. Chao, X. et al. A novel magnetic nanoparticle drug carrier for enhanced cancer chemotherapy. PLoS One. 7, e40388 (2011).

15. Liu, X. et al. Enhanced retention and cellular uptake of nanoparticles in tumors by controlling their aggregation behavior. ACS Nano. 7, 6244-6257 (2013).

16. Kutty, R. V. \& Feng, S. S. Cetuximab conjugated vitamin E TPGS micelles for targeted delivery of docetaxel for treatment of triple negative breast cancers. Biomaterials. 34, 10160-10171 (2013).

17. Ettinger, D. S. Clinical implications of EGFR expression in the development and progression of solid tumors: focus on non-small cell lung cancer. Oncologist. 11, 358-373. (2006)

18. Chen, Y. H. et al. Methotrexate conjugated to gold nanoparticles inhibits tumor growth in a syngeneic lung tumor model. Mol Pharm. 4, 713-722 (2007).
19. Huang, S. et al. Dual targeting of EGFR and HER3 with MEHD7945A overcomes acquired resistance to EGFR inhibitors and radiation. Cancer Res. 73, 824-833 (2013).

20. Wu, Y. N. et al. The anticancer properties of iron core-gold shell nanoparticles in colorectal cancer cells. Int J Nanomedicine. 8, 3321-3331 (2013).

21. Brown, S. D. et al. Gold nanoparticles for the improved anticancer drug delivery of the active component of oxaliplatin. J Am Chem Soc. 132, 4678-4684 (2013).

22. Pan, Y. et al. Gold nanoparticles of diameter $1.4 \mathrm{~nm}$ trigger necrosis by oxidative stress and mitochondrial damage. Small. 5, 2067-2076 (2009).

23. Prabaharan, M., Grailer, J. J., Pilla, S., Steeber, D. A. \& Gong, S. Gold nanoparticles with a monolayer of doxorubicin-conjugated amphiphilic block copolymer for tumor-targeted drug delivery. Biomaterials. 30, 6065-6075 (2009).

24. Takata, M. et al. Lack of AKT activation in lung cancer cells with EGFR mutation is a novel marker of cetuximab sensitivity. Cancer Biol Ther. 13, 369-378 (2012).

25. Mukherjee, P. et al. Antiangiogenic properties of gold nanoparticles. Clin Cancer Res. 11, 3530-3534 (2005).

26. Bhattacharyya, S. et al. Nanoconjugation modulates the trafficking and mechanism of antibody induced receptor endocytosis. Proc Natl Acad Sci USA 33, 14541-14546 (2010).

\section{Acknowledgments}

This work was supported by grants from Natural Science Foundation of Jiangsu Province (BK2011036), Research Grant of National Ministry of Health (2012-W6), Key Clinical Medicine Technology Foundation of Jiangsu Province (BL2012030), and National Natural Science Foundation of China (81201830, 81372321), China. The funders had no role in study design, data collection and analysis, decision to publish, or preparation of the manuscript.

\section{Author contributions}

Y.C.Q. and Q.Q.W. performed the main experiments and analysed the data. Y.Y.T., Y.Z. N.G. and S.Y.L. conducted the experiments to synthetize C225-AuNPs. Y.C.Q., M.T.Q.,

R.Y. and L.X. conceived of and designed experiments and wrote the manuscript.

\section{Additional information}

Competing financial interests: The authors declare no competing financial interests.

How to cite this article: Qian, Y. et al. Enhanced cytotoxic activity of cetuximab in EGFR-positive lung cancer by conjugating with gold nanoparticles. Sci. Rep. 4, 7490; DOI:10.1038/srep07490 (2014)

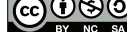

This work is licensed under a Creative Commons Attribution-NonCommercialShareAlike 4.0 International License. The images or other third party material in this article are included in the article's Creative Commons license, unless indicated otherwise in the credit line; if the material is not included under the Creative Commons license, users will need to obtain permission from the license holder in order to reproduce the material. To view a copy of this license, visit http:// creativecommons.org/licenses/by-nc-sa/4.0/ 